\title{
Finite element analysis of surface modes in phononic crystal waveguides
}

\author{
Yuning Guo, ${ }^{\text {a) }}$ Martin Schubert, and Thomas Dekorsy \\ Department of Physics and Center of Applied Photonics, University of Konstanz, 78457 Konstanz, Germany
}

\begin{abstract}
The study of surface modes in phononic crystal waveguides in the hypersonic regime is a burgeoning field with a large number of possible applications. By using the finite element method, the band structure and the corresponding transmission spectrum of surface acoustic waves in phononic crystal waveguides generated by line defects in a silicon pillar-substrate system were calculated and investigated. The bandgaps are caused by the hybridization effect of band branches induced by local resonances and propagating modes in the substrate. By changing the sizes of selected pillars in the phononic crystal waveguides, the corresponding bands shift and localized modes emerge due to the local resonance effect induced by the pillars. This effect offers further possibilities for tailoring the propagation and filtering of elastic waves. The presented results have implications for the engineering of phonon dynamics in phononic nanostructures.
\end{abstract}

\section{INTRODUCTION}

In recent years, advances in nanotechnology have enabled the fabrication of nanophononic and nanomechanical systems. These systems have emerged as intriguing subjects for studying mechanics, heat transfer, and optomechanical coupling, which have opened exciting possibilities to control phonons. Periodic nanostructures exhibit several novel features in coherent electronic, photonic, thermal, as well as phononic transport. Novel phenomena have been investigated, for example, enhanced light-matter interaction, ultrafast acoustoplasmonic control, and sensing by evanescent surface acoustic waves (SAWs). ${ }^{1-4}$ Phononic nanostructures could lead to a variety of potential applications, ranging from acoustic metamaterials, self-collimation, sound isolation, and heat management to compact acoustic waveguides. ${ }^{5-9}$ Similar to their photonic counterparts, the flexibility to tailor the acoustic properties of phononic crystals (PnCs) and $\mathrm{PnC}$ waveguides makes them particularly suitable for a wide range of applications from transducer technology to filtering, guiding, and demultiplexing of acoustic waves. The accelerated advances in fabrication have allowed the reduction of the characteristic sizes of PnC down to the nm scale, enabling the control of phonons in the $\mathrm{GHz}$ frequency range. ${ }^{10,11}$

Several calculation methods have been developed for the design of nanostructures and their optimization. They include plane wave expansion (PWE), the transfer matrix method (TMM), multiple scattering theory (MST), finitedifference time-domain (FDTD), and the finite element method (FEM). ${ }^{12-16}$ Effects, such as band folding, mode leakage, opening of frequency gaps, and interaction between slab and overlayer modes, have been studied by simulation. ${ }^{17-19}$ SAWs can be conveniently excited and detected by piezoelectric material or optical approaches. SAW-based components are extensively used as radio-frequency filters in modern communication systems. Especially, high frequency

\footnotetext{
a) Author to whom correspondence should be addressed. Electronic mail: yuning.guo@uni konstanz.de
}

SAWs have found applications in material characterization, photonic modulation, phononic sensor, optomechanics, and transport by phonons or other excitations in solids. ${ }^{20,21}$ Periodic nanostructures are used to modify the properties of SAWs, perturbing the stress and velocity fields associated with SAW propagation. ${ }^{22,23}$ The published studies show that the filling factor, the ratio of the slab thickness to the lattice period, and the height of the pillar are the key parameters for the existence of complete bandgaps. ${ }^{24,25}$ When the substrate's surface acoustic modes strongly couple to the periodic overlayer, the SAW will evolve into a pseudo-SAW which is partially localized in the nanostructures and radiates mechanical energy into the substrate due to the scattering introduced by the periodic overlayer. ${ }^{26,27}$ Basic surface modes can be recognized due to their relatively lower velocity compared to bulk wave's in solids in some situations. By using a sound cone limitation, the propagation of surface acoustic waves in different types of phononic structures and the SAW bandgap for piezoelectric phononic crystals in honeycomb lattice are investigated. ${ }^{28,29}$ The bandgap induced by local resonances, which can be tuned by changing the characteristic size of the pillars, has been studied by Pennec et ll $^{30}$ Afterwards, Khelif et al. studied a PnC composed of silicon pillars on a silicon substrate and obtained the bandgaps of locally resonant surface acoustic waves. ${ }^{31}$ The experiments of SAW band gaps also have been investigated in nanoscale 2D pillars system and surface phonon-polariton phononic crystals. ${ }^{32,33}$ Defects in PnCs can localize the elastic wave at the point defect or it will propagate along line defects. Beside the bandgap, band edge states also exhibit some interesting phenomena worth to be investigated, such as bending of acoustic waves, strong dispersion of the group velocity, and the superlens phenomenon. Experiments have directly demonstrated the trapping and guiding of surfaceguided elastic waves in linear defects in a micron-scale phononic crystal. ${ }^{34}$

Based on the calculations of phononic characteristics in the finite size PnC model, the band modulation in $\mathrm{PnC}$ 
waveguides is investigated in this paper. This study demonstrates that localized modes can be enhanced in the $\mathrm{GHz}$ phononic structures, which is potentially attractive for highfrequency applications. The paper is organized as follows. In Section II, we summarize the essentials of our calculation method and introduce the calculation models; Section III is devoted to the analysis and discussion of band structure and transmission spectra of phononic structures: the first part contains the surface modes analysis of PnC; the second part is the main result of this paper about the surface modes in line-defects PnC waveguides; and Section IV contains the conclusion.

\section{MODEL AND METHOD OF CALCULATION}

PnC waveguides offer the possibility to tailor the dispersion properties while the cavities are used for achieving confinement. The acoustic equation of motion for the composite system is described as

$$
\rho(\mathbf{r}) \ddot{u}_{i}=\partial_{j}\left[c_{i j m n}(\mathbf{r}) \partial_{n} u_{m}\right], \quad(i=x, y, z),
$$

where $\mathbf{u}(\mathbf{r}, t)$ is the lattice displacement, $\rho(\mathbf{r})$ and $c_{i j m n}(\mathbf{r})$ are the position-dependent mass density and elastic stiffness tensor, respectively, and the summation convention over repeated indices is assumed. The pillar-substrate system is an appropriate system to investigate the phononic characteristics for practical applications. The band structure and transmission spectrum of SAWs in the line-defects of a PnC waveguide are analysed by using Comsol Multiphysics which is based on FEM. We present a PnC consisting of a square array of cylindrical silicon pillars on a silicon substrate in air. By variation of structural parameters and defects, frequencies characteristics can be tailored, demonstrating the structure's ability to be used as a frequency filter.
Figure 1 shows the scheme of the phononic structures studied in this paper. The pillars are oriented perpendicular to the [100] direction on the (001) surface of silicon. For the calculation of the band structure of $\mathrm{PnC}$, a unit cell is used as shown in Fig. 1(a). The height of pillar $h_{1}$, radius of pillar $r$, and lattice parameter $a$ are $100 \mathrm{~nm}, 65 \mathrm{~nm}$, and $200 \mathrm{~nm}$, respectively. The filling factor $f$ is defined as $f=\pi r^{2} / a^{2}$. To decouple the Lamb wave from surface modes, thick plates larger than several lattice periods are considered. Taking into account the fact that the penetration depth of SAWs is roughly onewavelength from the surface, and after several tests, we found that the simulation results change little when the thickness is more than five times the lattice parameter. Thus, to reduce the computation time, the thickness of the layer accounting for the substrate is taken to be five times the lattice constant $h=5^{*} a$ throughout this paper. Taking into account the anisotropy of silicon, calculations are performed with elastic constant $\mathrm{C}_{11}=165.7 \mathrm{GPa}, \mathrm{C}_{12}=63.9 \mathrm{GPa}, \mathrm{C}_{44}=79.9 \mathrm{GPa}$, and mass density $\rho=2331 \mathrm{~kg} / \mathrm{m}^{3}$ in this paper. Fixed boundary conditions are adopted for the bottom of the structure, free boundary condition for the upper surface, and periodic boundary conditions (PBCs) for the interfaces between the nearest unit cells according to Bloch-Floquet theorem. The Bloch-Floquet theorem can be written as

$$
u_{i}(x+a, y+a)=u_{i}(x, y) \exp \left(-i\left(k_{x} a+k_{y} a\right)\right)
$$

where $i=x, y$, and $k_{x}$ and $k_{y}$ are Bloch wave vectors. By sweeping the wave vector in the first Brillouin zone and solving the eigenfrequency equation, the band structure and the vibrational modes of the structure are obtained. To elaborate the frequency characteristic of $\mathrm{PnC}$, the square lattice array model consisting of eleven periods is used and the transmission spectrum along the $x$ direction is described as shown in Fig. 1(b). Perfectly matched layers (PMLs) are applied along (a)

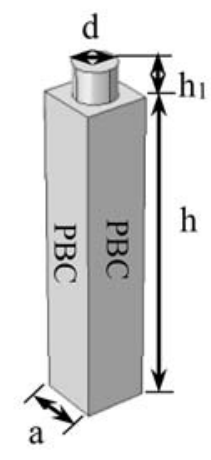

(c)

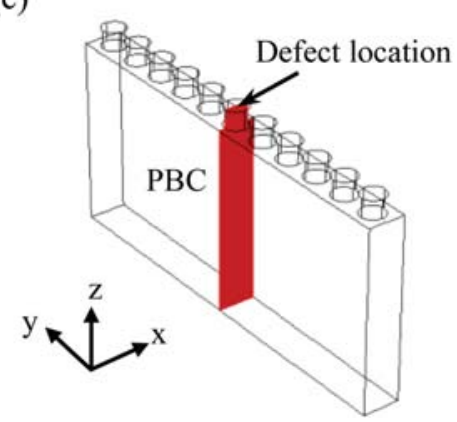

(b)

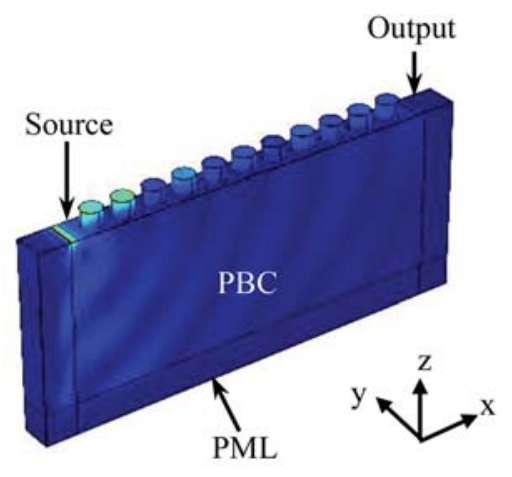

(d)

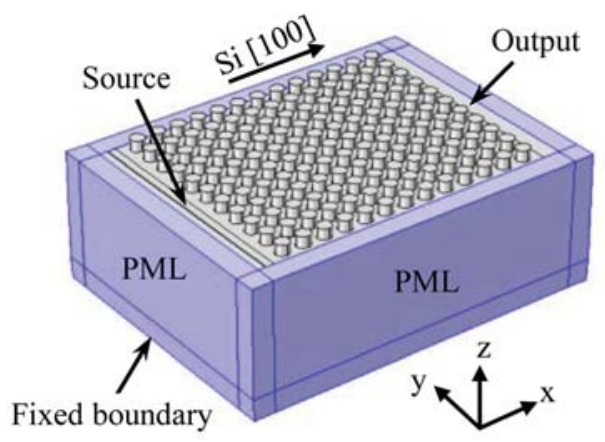

FIG. 1. Scheme of the phononic struc ture models: (a) the unit cell of $\mathrm{PnC}$ composed of silicon pillars on a sub strate arranged in a square array; (b) the transmission model of $\mathrm{PnC}$ in $x$ direction, the perfectly matched layers (PMLs) are applied in both sides and periodic boundary conditions are set along y direction; (c) the model for band structure calculations of $\mathrm{PnC}$ waveguide, periodic boundary condi tions (PBCs) are set along the $x$ direc tion. The size of the middle pillar is changed as defect; the red facet is a 2D cross section; (d) the transmission model of PnC waveguides (finite size PnCs with defects). 

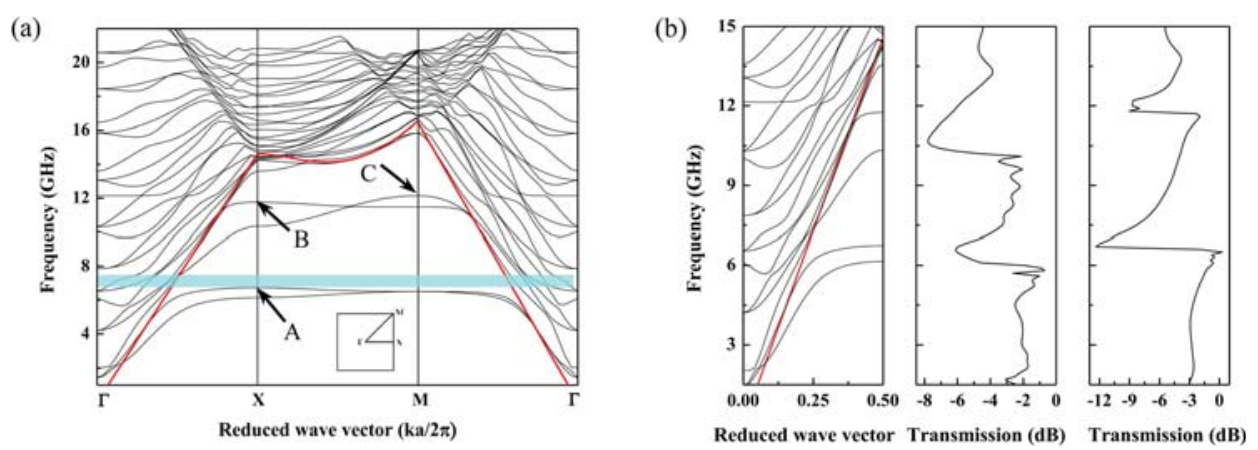

FIG. 2. (a) Band structures of PnC: the red line here is the sound cone, the blue horizontal bar represents the location of the bandgap, the points (A C) indicate the frequency and wavevector of the modes depicted in Fig. 3, The inset shows the first irreducible Brillouin zone of a square lattice. (b) Left: dispersion rela tion in $\Gamma X$ direction, middle: the corresponding transmission spectrum with sagittal polarized excitation line source, right: the corresponding transmission spectrum with shear horizontally polarized excitation line source.

the $x$ direction and the bottom to avoid backscattering from the boundaries. PBCs are applied to the front and back boundaries, which are along the $y$ direction. A line source is applied at the surface of the silicon substrate in front of the phononic structure. The excitation source can have two polarizations: $\left(u_{\mathrm{x}}, u_{\mathrm{z}}\right)$ sagittal displacement which excites Rayleigh surface waves; $u_{\mathrm{y}}$ transverse displacement which can be considered as a shear horizontal wave source. ${ }^{31}$ The output signal is integrated at the location over the cross section of the right boundary of the phononic in the $y$ direction. The transmissions spectrum represents the sum of the square of three displacement components, $u_{\mathrm{x}}{ }^{2}+u_{\mathrm{y}}{ }^{2}+u_{\mathrm{z}}{ }^{2}$ as a function of frequency, which can be regarded as energy transmission spectrum.

To understand the properties of surface modes in linedefect phononic waveguides, the model of square lattice array in finite size is used to analyse the dispersion properties of the PnC waveguides with different defects. The guided modes in the phononic waveguides with linear defect are formed by removing one row of cylinders or by changing the radius constituting the pillars in a row. As shown in Fig. 1(c), a square lattice array model containing eleven unit cells is used. The size of the sixth pillar is changed as defect. The size of the defect is set as $0 \mathrm{~nm}, 40 \mathrm{~nm}, 65 \mathrm{~nm}$, and $85 \mathrm{~nm}$ to form waveguides. The facet coloured in red in Fig. 1(c) is the two-dimensional cross section of the model, which is used to investigate the mode characteristic of the defect. To calculate the transmission spectrum, the sagittal polarized excitation line source and the shear horizontally polarized excitation line source are set as input signals as shown in Fig. 1(d). PMLs are applied all around the structure and at the bottom. Beside SAWs in the upper surface, the SAW propagation in the surface which is perpendicular $y$ direction has also been studied. ${ }^{8,35}$ As the surface modes in that surface are not the research point in this paper, the boundaries are fixed to avoid interference.

\section{RESULTS AND DISCUSSION}

\section{A. Surface mode analysis of PnC}

Figure 2 gives the dispersion relations of the silicon pillar-typed phononic crystal based on the parameters given in the previous chapter. The irreducible Brillouin zone, shown in the inset of Fig. 2, depends on the symmetry of the phononic crystal lattice and isotropy of the material. In the band structure of the PnC, the first order derivative of the frequency over the wave vector is the group velocity. The red solid line represents the sound cone of silicon which allows distinguishing the surface acoustic modes. The sound line here corresponds to the lowest bulk wave velocity in silicon. Those modes below the sound cone which are confined in the PnC are of interest to us. A frequency gap, which spans from $6.8 \mathrm{GHz}$ to $7.5 \mathrm{GHz}$, is observed, where the acoustic wavelength is almost four times larger than the size of the lattice constant. The centre frequency of the lowest bandgap in the PnC induced by Bragg scattering is at about $u / 2 a, u$ is the velocity and $a$ is the lattice constant, the corresponding wavelength is twice of the lattice constant, so this bandgap is not induced by Bragg scattering. ${ }^{21}$ Based on the analysis in Ref. 31 , we attribute this bandgap to the interaction of normal acoustic band branches and a flat band produced by the local resonance of the phononic structure, which means that the resonant modes of the pillars interact with surface modes of the substrate to open bandgaps. The calculation of transmission spectra can depict a clear picture of the band structure. Fig. 2(b) shows the transmission spectra for the sagittal and the shear horizontally polarized line source which correspond to the band structure in $\Gamma \mathrm{X}$ direction. Energy transmissions with the frequency of the sagittal polarized source and the shear horizontally polarized source both agree with the dispersion curves. For the transmission with the sagittal line source, two attenuation ranges correspond to the first and the third band. While with a shear horizontally line source, the attenuation range corresponds well to the second and fourth bands.

The guided surface modes are localized inside the sound cone while the bulk modes are outside. To clearly demonstrate the nature of the dispersion relations inside the sound cone, an eigenmode analysis is performed and displacement fields are computed. Relative values of the displacement fields and the corresponding displacement components for the guided surface modes as indicated in the band structure in Fig. 2(a) are shown in Fig. 3. The displacement fields of points $\mathrm{A}, \mathrm{B}$, and $\mathrm{C}$ are at the frequency of $6.7 \mathrm{GHz}$, 10.34 GHz, and $12.13 \mathrm{GHz}$, respectively.

As shown in Fig. 3, the elastic energy is mostly confined in the pillar and in the vicinity area of substrate's 


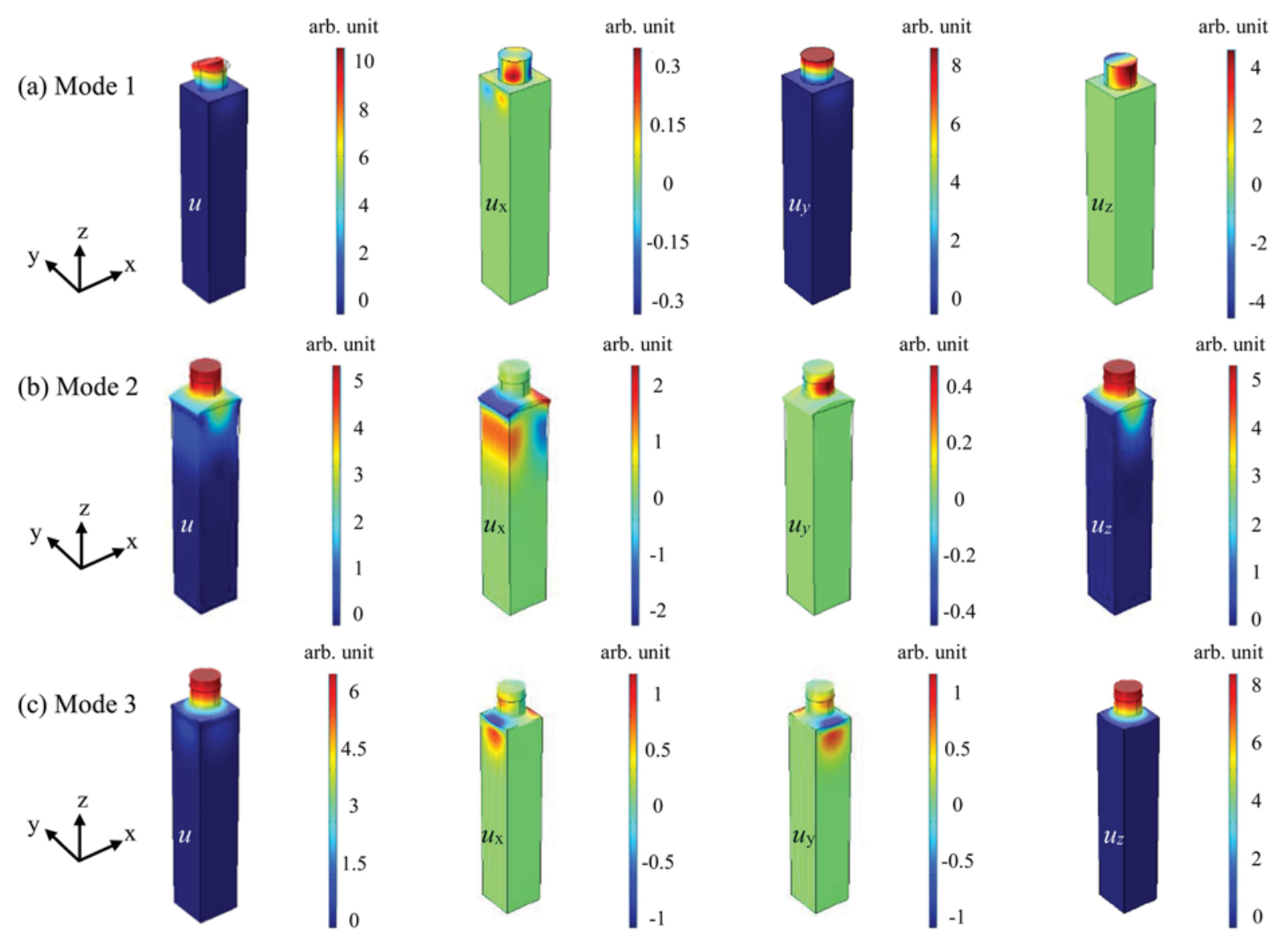

FIG. 3. Displacement fields for the surface modes indicated in Fig. 2(a), from left to right are the total displacement $u$, the displacement components $u_{\mathrm{x}}, u_{\mathrm{y}}$, and $u_{\mathrm{z}}$, respectively. (a) Mode 1: point $\mathrm{A}$, frequency at $6.7 \mathrm{GHz}$, located in the second band. (b) Mode 2: point $\mathrm{B}$, frequency at $10.34 \mathrm{GHz}$, located in the third band. (c) Mode 3: point $\mathrm{C}$, frequency at $12.13 \mathrm{GHz}$, located in the fourth band.

surface which is the characteristic of a surface mode. In a periodically patterned surface, the SAW is characterized by different polarizations, dispersive, and localization features $^{29}$ which are more complex than those of classical surface waves propagating on a homogeneous surface. From this figure, we estimate that the displacement field of a guided mode confined within a depth equal to the wavelength from the top surface, which agrees with Ref. 27. Beside the energy transferred to the pillar, the proportion of energy transferred into the substrate varies obviously. Mode 1 has a shear oscillation, and modes 2 and 3 have elongational oscillations. As Fig. 3(a) shows, the energy of mode 1 is concentrated in the pillar, particularly, $u_{\mathrm{z}}$ owns an antisymmetric character (the vibration directions of displacement in the pillar are opposite) with respect to the sagittal midplane $(x, z)$ of the structure which could explain the absence of response of certain bands (second and fourth bands here) in the transmission spectra with the sagittally polarized source. ${ }^{31}$ The surface mode shown in Fig. 3(b) is mostly sagittal polarized which agrees with the transmission spectra for the sagittal polarized source. The acoustic energy is mostly distributed between $u_{\mathrm{x}}$ and $u_{\mathrm{z}}$, while $u_{\mathrm{y}}$ is relatively small. Point $C$ is shown for comparison; the three displacement components all make an approximately equal contribution to the displacement field. The mode analysis also confirms that resonant modes of the pillars can interact with the surface modes of the substrate.
The band structures for different pillar radii are calculated for comparison. Except the radius of pillar, all other parameters are the same as in Fig. 1(b). As shown in Fig. 4, the bandgap with a pillar radius of $40 \mathrm{~nm}(f=0.13)$ is smaller and the centre frequency is lower, while the bandgap with pillar radius of $85 \mathrm{~nm}(f=0.57)$ is larger and the centre frequency is higher. Based on our simulations, we find that the width of the bandgap increases and the centre frequency of the bandgap is pushed down towards higher frequency with increasing filling factor from 0.07 to 0.78 in such a structure. By increasing the radius of the pillar, the interaction between locally resonant modes is enhanced by surface coupling which in turn leads to a larger bandgap.

\section{B. The surface modes analysis of line-defects PnC waveguide}

To understand the properties of surface modes in linedefects phononic waveguides, the square lattice array in finite size PnC is used to analyse the dispersion properties of the waveguide. The guided modes in the phononic waveguides with linear defect are analysed in this chapter.

Figure 5 shows the phononic band structures and the corresponding transmission spectra of line-defects $\mathrm{PnC}$ waveguides with a sagittal polarized excitation line source and a shear horizontally polarized excitation line source. By changing the size of the defect, the characteristics of the 


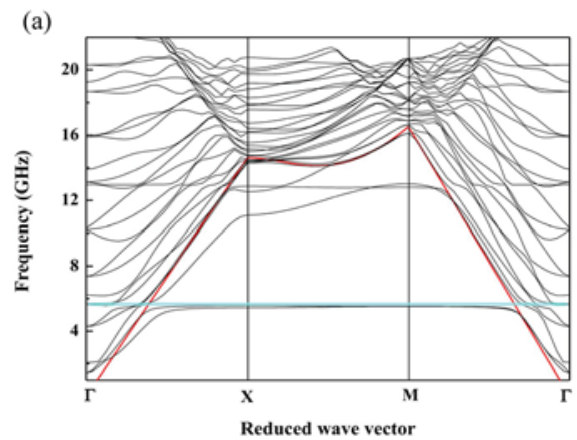

(b)

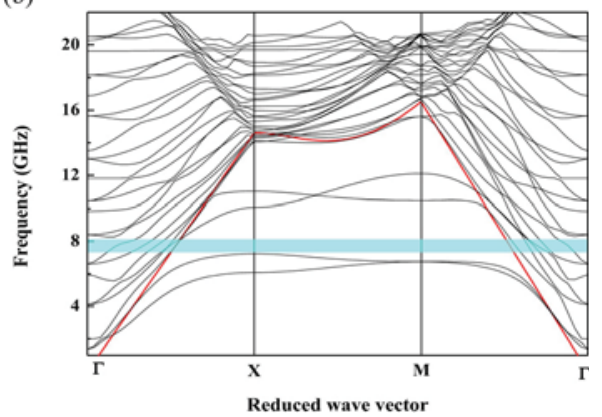

FIG. 4. Band structures of $\mathrm{PnC}$ (a) with a pillar radius of $40 \mathrm{~nm}$; (b) with a pillar radius of $85 \mathrm{~nm}$. The blue hori zontal bar represents the location of the bandgap. band structure, the frequency spectrum, and the elastic wave modes are investigated. As shown in Fig. 5, the locations of bandgap change slightly with different defect size. The transmission spectra agree with the corresponding band structure. With an increase of the radius of defect, the transmission peak of the sagittal polarized line source shifts gradually to lower frequency, indicating an energy transfer to lower frequencies. The transmission peak of the shear horizontally polarized line source shows no such trend. The solutions of FEM calculations with higher frequencies are not shown here since they do not satisfy the criteria of surface modes. Comparing Fig. 5(c) with Fig. 2(b), it can be seen that the band structure of the finite size PnC is much more complex than that of a single unit cell, which is caused by the finite size in $y$ direction that produces constraint to the band superposition in the finite size PnC. While they still have similar features, such as the locations and widths of bandgaps are the same, some bands are in the same frequency range. Although there is no large difference in the overall band structures at four conditions, some bands change the location which is worthy to be studied. It is evident that the first band and the second band decline in Fig. 5(b), while a band appears in the bandgap region in Fig. 5(d). These three bands support modes that are well confined in the pillars which are (a)

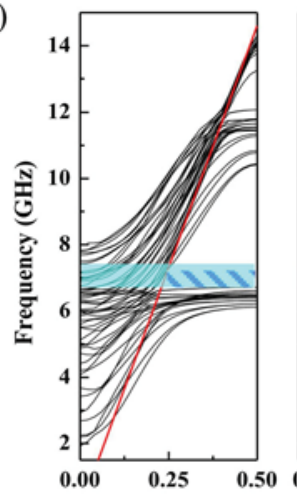

Reduced wave vector

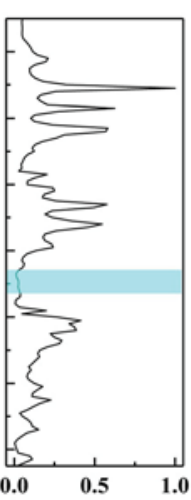

Transmission

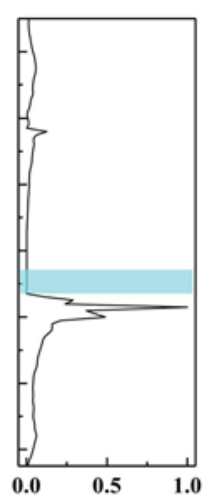

Transmission (c)

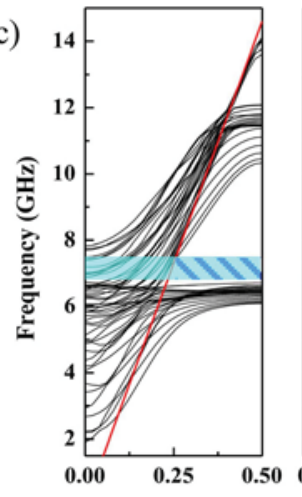

Reduced wave vector
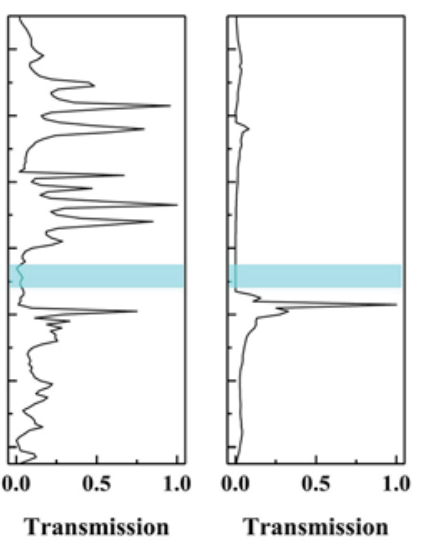

(b)
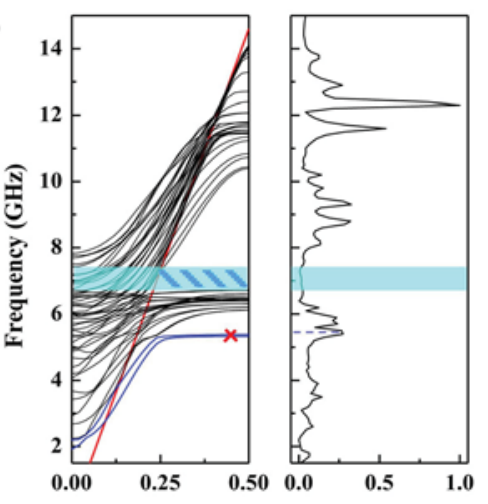

Reduced wave vector

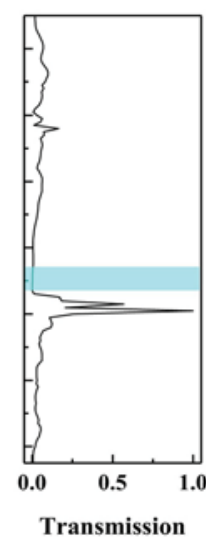

(d)

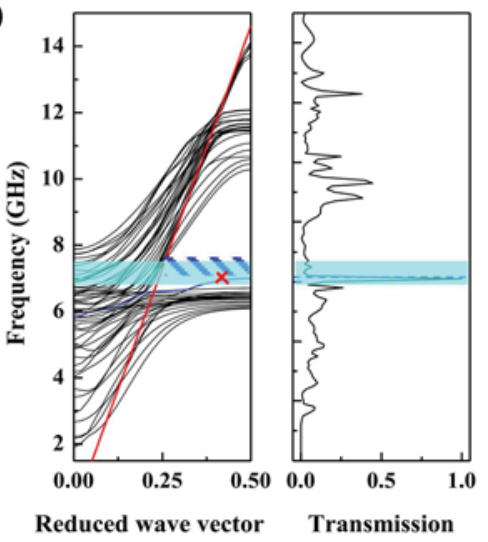

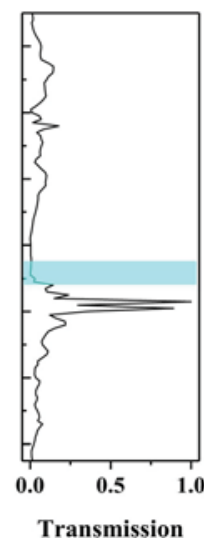

FIG. 5. Each subfigure shows the band structures (left), the corresponding normalized transmission spectrum with a sagittal polarized excitation line source (middle) and the transmission spectrum with a shear horizontally polarized excitation line source (right) of PnC waveguides with varied defect radii in a square array. (a) Defect radius $r \quad 0$ (conventional line defect waveguide); (b) with defect radius $r \quad 40 \mathrm{~nm}$; (c) with defect $r \quad 65 \mathrm{~nm}$, perfect PnC; (d) with defect ra dius $r \quad 85 \mathrm{~nm}$. The blue horizontal bar represents the location of the bandgap of the perfect PnC. The patterned area is the location of a bandgap at the condi tion with defect. The red crosses mark the shifted bands. 
shown in Fig. 6. After checking the concrete bands of perfect $\mathrm{PnC}$ and larger defect waveguides, we find that the locations of the 20th band and the 22nd band are almost the same, only the $21 \mathrm{st}$ band shifts at defect radius $\mathrm{r}=85 \mathrm{~nm}$ condition. Therefore, we think the 21st band (marked as red cross in Fig. 5(d)) is not a new band introduced by the defect, but a shifted band. Based on the analysis of Fig. 4, we know that larger defects lead to a frequency increase of a band and bandwidth. Hence, when a large defect exists in the finite size $\mathrm{PnC}$, it has a similar effect on the superposition bands as Fig. 5(d) shows. As shown in Fig. 5(b), a small defect has the opposite effect which decreases the bands and narrows the bandgap. Therefore, the larger defect in finite size PnC makes certain modes increase in frequency while smaller defects shift certain modes to lower frequencies.

The defects can strengthen the localized states in the band structure, which also causes certain bands to shift. The frequency of the shifted band (marked as red cross in Fig. 5) is the local resonance frequency which is determined by the defect size. Figure 5(d) evidently shows that the transmission at $7 \mathrm{GHz}$ (the rising band marked by a red cross) with the sagittal polarized line source increases significantly, which can be explained due to the frequency of the rising band located in the bandgap range of the perfect PnC. This band in the bandgap causes the distribution of the transmission spectrum to change obviously, i.e., a defect inside the bandgap permits to select a particular frequency to be transmitted intensively. Hence, for the small defect shown in Fig. 5(b), the transmission intensity does not change very much at the frequency of the declining bands as these two bands sit at the edge of the band structure, but not in the bandgap. By changing the defect size, the locations of certain bands can be tailored to rise or decline in $\mathrm{PnC}$ waveguide, which allows to separate or filter the corresponding frequencies.

As shown in Figs. 5(a) and 5(c), the distinction of band structures between perfect $\mathrm{PnC}$ and conventional line defect waveguide is not evident, no band appears in the bandgap or shifts obviously in the conventional line defect waveguide. This can be explained due to the bandgap being influenced by the resonance frequency associated with the parameters of a resonator, which depends less on the periodicity and the symmetry of the structure. That is to say, the linear waveguide does not introduce new localized resonance mode to the band structure, so no band shift or appear obviously in comparison to the perfect $\mathrm{PnC}$ condition. We also confirm that there are no similar characteristics in band structures and transmission spectrum in hole-type $\mathrm{PnC}$ when the size of defects is changed. It is due to the fact that hole-tape PnC does not produce a local resonance effect, which confirms the above analysis from another aspect. Other interesting modes are guided modes with flat dispersion, including bands around $6.7 \mathrm{GHz}$ for all conditions and the two shifted bands in Fig. 5(b), which own special characteristics (a)

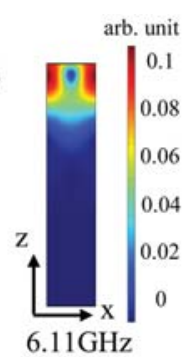

(c)

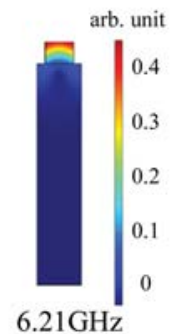

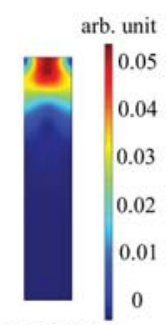

$6.72 \mathrm{GHz}$

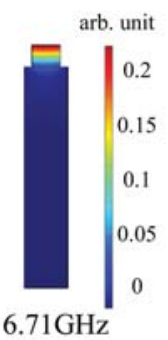

(e)

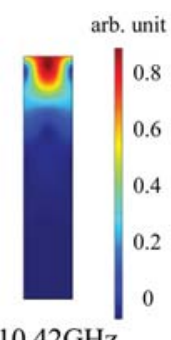

$10.42 \mathrm{GHz}$

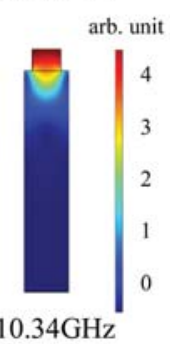

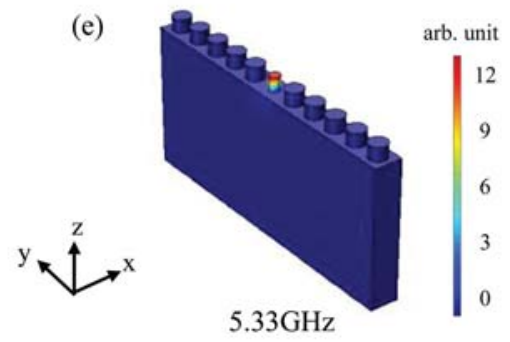

(b)

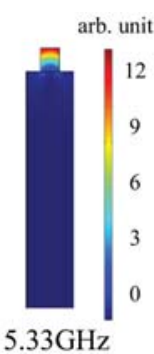

(d)

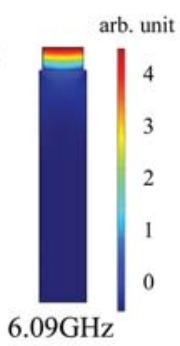

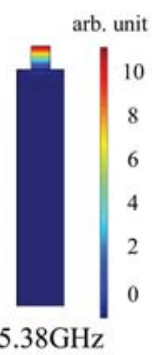

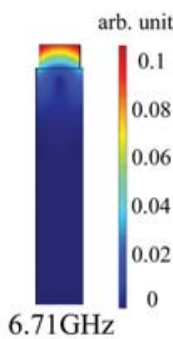

$6.71 \mathrm{GHz}$

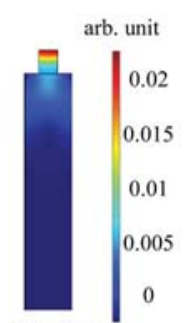

$6.72 \mathrm{GHz}$

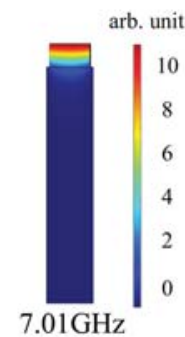

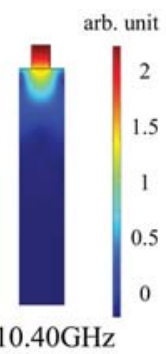

arb, unit

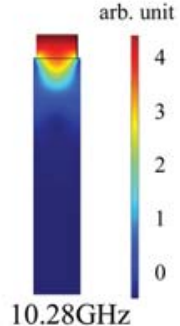

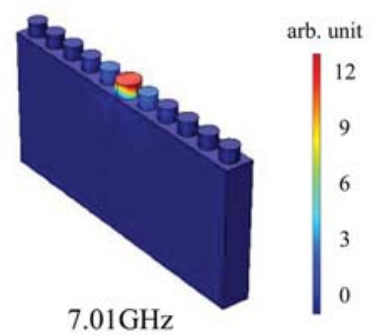

FIG. 6. The displacement fields of guided waves in PnC waveguides. (a) (d) The modes of the $2 \mathrm{D}$ cross section in the $\mathrm{X}$ point $(\mathrm{ka} / 2 \pi \quad 0.5)$ at different eigen frequencies. (a) Linear waveguide: modes frequency at $6.11 \mathrm{GHz}, 6.72 \mathrm{GHz}$, and $10.42 \mathrm{GHz}$. (b) With small defect r $40 \mathrm{~nm}$ : modes frequency at $5.33 \mathrm{GHz}$, $5.38 \mathrm{GHz}, 6.72 \mathrm{GHz}$, and $10.40 \mathrm{GHz}$. (c) PnC: modes frequency at $6.21 \mathrm{GHz}, 6.71 \mathrm{GHz}$, and $10.34 \mathrm{GHz}$. (d) With large defect r $85 \mathrm{~nm}$ : modes frequency at $6.09 \mathrm{GHz}, 6.71 \mathrm{GHz}, 7.01 \mathrm{GHz}$, and $10.28 \mathrm{GHz}$. (e) 3D model of localized modes, with small defect: $5.33 \mathrm{GHz}$; with large defect: $7.01 \mathrm{GHz}$. 
introduced below. The PnC waveguides show the ability to strengthen the guided modes with respect to the Si membrane. Localized resonant mode gives rise to a flat band which shows zero group velocity, the elastic energy is completely or mostly confined within the resonator. The mode analysis of SAWs in PnC waveguide with varied defect radius is discussed as follows.

Figs. 6(a) 6(d) describe the displacement fields of twodimensional cross sections (red facet in Fig. 1(c)) of different models at different eigenfrequencies in the $\mathrm{X}$ point $(\mathrm{ka} /$ $2 \pi=0.5$ ) in a linear waveguide, a waveguide with small defect $r=40 \mathrm{~nm}$, a perfect $\mathrm{PnC}$, and a waveguide with large defect $r=85 \mathrm{~nm}$, respectively. We can observe that the displacement fields of guided waves at different frequencies localize within pillars or the adjacent area of the substrate surface. As shown in Figs. 6(b) and 6(d), the shifted bands, including the 1 st and the 2 nd band under small defect condition and the band at $7.01 \mathrm{GHz}$ under large defect condition, exhibit strong localization. Those modes are mainly localized in the pillars with little deformation of the substrates. According to the former analysis, when the size of the defects is changed, certain branches rise or decline in the band structures. Each of them support a mode that is well confined within the pillar by the local resonance effect which can be clearly seen in Fig. 6(e). In addition, since flat bands also produce localized modes, the ones with flattest dispersion (lowest group velocity) also show the characteristics of local resonance at $6.7 \mathrm{GHz}$ as shown in Figs. 6(a) 6(d). The strongly localized mode here is the surface mode which becomes standing Bloch wave and exhibits a well local or non-propagating character induced by the resonance of pillar. The cos-SAW modes also can be seen in Figs. 6(a) 6(d). By introducing a defect of appropriate radius, the localized defect state can be strengthened in the phononic structure, which can be applied for wave filtering and demultiplexing.

\section{CONCLUSION}

The theoretical analysis of surface modes in line-defect $\mathrm{PnC}$ waveguides in the silicon pillar-substrate system in a square array is investigated in this paper. Confined surface modes induced by local resonance effect in the $\mathrm{GHz}$ range are analysed in detail. The bandgap is caused by the interaction of normal acoustic band branches and a flat band produced by the local resonances of the PnC structure, which demonstrate that the resonant modes of pillars interact with surface modes of the substrate and in turn open bandgaps. As the size of parts of the pillars changes in PnC waveguides, the corresponding bands will rise or decline hence forming strong localized modes due to the local resonance effect. This offers further possibilities for tailoring the propagation of elastic waves. The main conclusions are also valid for other material. The simulation results provide novel insight regarding $\mathrm{GHz}$ phonon dynamics in nanostructures. The discussed structures are potentially attractive for highfrequency applications. This paper provides the basis to predict the behaviour of high frequency SAW in PnC waveguides, which should facilitate to design technological useful structures and improve the understanding of high frequency surface acoustic waves in nanostructures in general.

\section{ACKNOWLEDGMENTS}

This work was supported by the German Research Foundation (DFG) through the SFB 767. Y. Guo gratefully acknowledges the financial support from the China Scholarship Council (CSC).

${ }^{1}$ A. H. Safavi Naeini, T. P. M. Alegre, J. Chan, M. Eichenfield, M. Winger, Q. Lin, J. T. Hill, D. E. Chang, and O. Painter, Nature 472, 69 (2011).

${ }^{2}$ K. O’Brien, N. D. Lanzillotti Kimura, J. Rho, H. Suchowski, X. Yin, and X. Zhang, Nat. Commun. 5, Article No. 4042 (2014).

${ }^{3}$ Y. Zhou, M. H. Lu, L. Feng, X. Ni, Y. F. Chen, Y. Y. Zhu, S. N. Zhu, and N. B. Ming, Phys. Rev. Lett. 104, 164301 (2010).

${ }^{4}$ B. L. Davis and M. I. Hussein, Phys. Rev. Lett. 112, 055505 (2014).

${ }^{5}$ Z. Liu, X. Zhang, Y. Mao, Y. Y. Zhu, Z. Yang, C. T. Chan, and P. Sheng, Science 289, 5485 (2000).

${ }^{6}$ M. H. Lu, L. Feng, and Y. F. Chen, Mater. Today 12, 34 (2009).

${ }^{7}$ D. M. Profunser, E. Muramoto, O. Matsuda, O. B. Wright, and U. Lang, Phys. Rev. B 80, 014301 (2009).

${ }^{8}$ B. Liang, B. Yuan, and J. C. Cheng, Phys. Rev. Lett. 103, 104301 (2009).

${ }^{9}$ M. Maldovan, Nature 503, 209 (2013).

${ }^{10}$ N. Zen, T. A. Puurtinen, T. J. Isotalo, S. Chaudhuri, and I. J. Maasilta, Nat. Commun. 5, Article No. 3435 (2014)

${ }^{11}$ D. Schneider, F. Liaqat, E. H. El Boudouti, Y. El Hassouani, B. Djafari Rouhani, W. Tremel, H. J. Butt, and G. Fytas, Nano Lett. 12, 3101 (2012).

${ }^{12}$ J. O. Vasseur, P. A. Deymier, B. Chenni, B. Djafari Rouhani, L. Dobrzynski, and D. Prevost, Phys. Rev. Lett. 86, 3012 (2001).

${ }^{13}$ Z. Y. Li and K. M. Ho, Phys. Rev. B 68, 155101 (2003).

${ }^{14}$ L. M. Li and Z. Q. Zhang, Phys. Rev. B 58, 9587 (1998).

${ }^{15}$ Y. Tanaka, Y. Tomoyasu, and S. I. Tamura, Phys. Rev. B 62, 7387 (2000).

${ }^{16}$ C. Goffaux and J. Sánchez Dehesa, Phys. Rev. B 67, 144301 (2003).

${ }^{17}$ A. A. Maznev and O. B. Wright, J. Appl. Phys. 105, 123530 (2009).

${ }^{18}$ D. M. Profunser, O. B. Wright, and O. Matsuda, Phys. Rev. Lett. 97, 055502 (2006).

${ }^{19}$ T. C. Wu, T. T. Wu, and J. C. Hsu, Phys. Rev. B 79, 104306 (2009).

${ }^{20}$ T. J. Kippenberg and K. J. Vahala, Science 321, 1172 (2008).

${ }^{21}$ Y. Pennec, V. Laude, N. Papanikolaou, B. Djafari Rouhani, M. Oudich, S. El Jallal, C. B. Jean, M. E. Jose, and A. Martínez, Nanophotonics 3, 413 (2014).

${ }^{22}$ C. Giannetti, B. Revaz, F. Banfi, M. Montagnese, G. Ferrini, F. Cilento, S. Maccalli, P. Vavassori, G. Oliviero, E. Bontempi, L. E. Depero, V. Metlushko, and F. Parmigiani, Phys. Rev. B 76, 125413 (2007).

${ }^{23}$ H. N. Lin, H. J. Maris, L. B. Freund, K. Y. Lee, H. Luhn, and D. P. Kern, J. Appl. Phys. 73, 37 (1993).

${ }^{24}$ S. Yankin, A. Talbi, Y. Du, J. C. Gerbedoen, V. Preobrazhensky, P. Pernod, and O. B. Matar, J. Appl. Phys. 115, 244508 (2014).

${ }^{25}$ Q. Rolland, S. Dupont, J. Gazalet, J. C. Kastelik, Y. Pennec, B. Djafari Rouhani, and V. Laude, Opt. Express 22, 16288 (2014).

${ }^{26}$ D. Nardi, F. Banfi, C. Giannetti, B. Revaz, G. Ferrini, and F. Parmigiani, Phys. Rev. B 80, 104119 (2009).

${ }^{27}$ D. Nardi, M. Travagliati, M. E. Siemens, Q. Li, M. M. Murnane, H. C. Kapteyn, G. Ferrini, F. Parmigiani, and F. Banfi, Nano Lett. 11, 4126 (2011).

${ }^{28}$ D. Yudistira, Y. Pennec, B. D. Rouhani, S. Dupont, and V. Laude, Appl. Phys. Lett. 100, 061912 (2012).

${ }^{29}$ M. B. Assouar and M. Oudich, Appl. Phys. Lett. 99, 123505 (2011).

${ }^{30}$ Y. Pennec, B. Djafari Rouhani, H. Larabi, J. O. Vasseur, and A. C. Hladky Hennion, Phys. Rev. B 78, 104105 (2008).

${ }^{31}$ A. Khelif, Y. Achaoui, S. Benchabane, V. Laude, and B. Aoubiza, Phys. Rev. B 81, 214303 (2010).

${ }^{32}$ B. Graczykowski, S. Mielcarek, A. Trzaskowska, J. Sarkar, P. Hakonen, and B. Mroz, Phys. Rev. B 86, 085426 (2012).

${ }^{33}$ D. Yudistira, A. Boes, B. Djafari Rouhani, Y. Pennec, L. Y. Yeo, A. Mitchell, and J. R. Friend, Phys. Rev. Lett. 113, 215503 (2014).

${ }^{34}$ S. Benchabane, O. Gaiffe, R. Salut, G. Ulliac, V. Laude, and K. Kokkonen, Appl. Phys. Lett. 106, 081903 (2015).

${ }^{35}$ Y. Li, Z. Hou, M. Oudich, and M. B. Assouar, J. Appl. Phys. 112, 023524 (2012). 